\title{
\begin{tabular}{l|l} 
Mibraries & DSpace@MIT
\end{tabular}
}

\author{
MIT Open Access Articles
}

A thermodynamic basis for the electronic properties of molten semiconductors: the role of electronic entropy

The MIT Faculty has made this article openly available. Please share how this access benefits you. Your story matters.

Citation: Rinzler, Charles C., and A. Allanore. “A Thermodynamic Basis for the Electronic Properties of Molten Semiconductors: The Role of Electronic Entropy." Philosophical Magazine 97, 8 (December 2016): 561-571 (c) 2016 Informa UK limited, trading as Taylor \& Francis group

As Published: https://doi.org/10.1080/14786435.2016.1269968

Publisher: Taylor \& Francis

Persistent URL: http://hdl.handle.net/1721.1/114781

Version: Author's final manuscript: final author's manuscript post peer review, without publisher's formatting or copy editing

Terms of use: Creative Commons Attribution-Noncommercial-Share Alike 


\section{A Thermodynamic Basis for the Electronic Properties of Molten Semiconductors: The Role of Electronic Entropy}

Charles C. Rinzler ${ }^{2}$, A. Allanore ${ }^{1}$

${ }^{1}$ Massachusetts Institute of Technology, Department of Materials Science and Engineering

77 Massachusetts Avenue, Room 13-5066, Cambridge, MA, USA 02139

e-mail address: allanore@mit.edu

$617-452-2758$

${ }^{2}$ e-mail address: rinzler@mit.edu

617-314-1999 


\section{A Thermodynamic Basis for the Electronic Properties of Molten Semiconductors: The Role of Electronic Entropy}

The thermodynamic origin of a relation between features of the phase diagrams and the electronic properties of molten semiconductors is provided. Leveraging a quantitative connection between electronic properties and entropy, a criterion is derived to establish whether a system will retain its semiconducting properties in the molten phase. It is shown that electronic entropy is critical to the thermodynamics of molten semiconductor systems, driving key features of phase diagrams including, for example, miscibility gaps.

Keywords: entropy; electronic entropy; thermopower; molten semiconductor

PACS: $64,71,81$

\section{Introduction}

The study of the electronic properties of noncrystalline semiconductor systems has been a frontier in condensed matter physics and materials science since the 1960s [1,2]. Many of the tools developed by the solid state condensed matter community to describe and predict the electronic properties of semiconducting systems depend on the presence of long range order and the lack of strong electron correlation to define computationally tractable solutions to the equations of quantum mechanics. Despite the challenges associated with lack of long-range order on predicting the electronic properties of noncrystalline materials, much progress has been made to phenomenologically and qualitatively describe the basis of semiconducting properties of noncrystalline systems (e.g. amorphous solids and the liquid state).

However, the advances in the field over the past 50 years do not currently enable the determination of whether the liquid phase (i.e. the molten state) of a material will behave as a semiconductor. A correlation between certain features of the phase diagrams of systems that exhibit semiconductivity in the molten state has been discussed in the literature since the 1960s, and recent efforts to compile thermodynamic and electronic property data for those systems have confirmed the correlation [3]. However, no explanation for this correlation, nor any quantitative 
basis, has been proposed to enable the use of phase diagrams to predict the electronic properties of molten systems. We herein apply a quantitative theory connecting the electronic and thermodynamic properties of molten systems to substantiate this correlation. Fultz predicted that the study of the electronic entropy of high temperature systems would be valuable for the prediction of high temperature material properties [4]. In our previous work on molten semiconductors [5], we discussed the various contributions to entropy and demonstrated a quantitative connection between the electronic state entropy ${ }^{*}$, the electronic properties of molten systems, and their total mixing entropy. We herein leverage this connection to show how electronic entropy is at the origin of the qualitative correlation reported in the prior art.

\section{Properties of Molten Semiconductors}

A description of molten semiconductors is available in reference [5]. These systems exhibit semiconducting properties in the molten state. Many sulfides, oxides, tellurides, selenides, and other systems behave as semiconductors in the molten state. These systems have been studied for over 50 years $[1,2]$. However, there is as yet no model that provides reliable quantitative prediction of the electronic or thermodynamic properties of these systems, nor whether a system will behave as a semiconductor in the molten state.

As early as 1969 a correlation between certain features of phase diagrams of systems that exhibit solid state semiconductor compounds and molten semiconductivity was reported in the literature, as illustrated in Figure 1 [6].

\footnotetext{
* The reader is invited to consult reference 5 for a discussion on electronic entropy. Electronic entropy is comprised of configurational electronic entropy (the entropy associated with localized electrons) and the electronic state entropy (the entropy associated with the size of the accessible state space of electrons in the system). Electronic state entropy represents the contribution of delocalized electrons as they manifest in the density of states near the Fermi level. This entropy proves to be quantitatively related to electronic transport properties as shown in reference 5 .
} 
It was observed that systems that remain semiconductors (SC) in the molten state (SC to SC transition) tend to exhibit molten-phase miscibility gaps and congruent melting of a solid semiconductor compound. This has held true across dozens of systems that we have analyzed. Explanations for this observation have historically been qualitative in nature $[3,6]$. We hereafter analyze this observation within the framework of macroscopic thermodynamics and demonstrate that electronic entropy drives the above-mentioned features of the phase diagrams of molten semiconductors.

\section{Connection Between Features of Phase Diagrams and Electronic Properties}

The enthalpies and entropies of fusion of congruent melting compounds for some systems that exhibit semiconductor-to-semiconductor (SC-SC) and semiconductor-to-metal (SC-M) transitions at melting are shown in Figure 2 in a format analogous to that presented by Iida and Guthrie for elements [7].

Compounds melting to a molten semiconductor (SC-SC) exhibit enthalpies of fusion between 9 and $20 \mathrm{~kJ} \mathrm{~mol}^{-1}$, while those melting as a metallized liquid (SC-M) exhibit enthalpies of fusion between 24 and $60 \mathrm{~kJ} \mathrm{~mol}^{-1}$.

A clear separation of the enthalpies and entropies of fusion of compounds of different classes of material systems indicates the potential for defining a rule analogous to Richard's rule [4]. The slope of the line fit to the SC-SC data in Figure 2 is $10.0 \mathrm{~J} \mathrm{~mol}^{-1} \mathrm{~K}^{-1}$, the average entropy of fusion for molten semiconductor systems predicted by Richard for metallic systems $\left(9.6 \mathrm{~J} \mathrm{~mol}^{-1} \mathrm{~K}^{-1}\right)$ [8]. Semiconductor compounds that do not change their electronic behavior upon melting exhibit similar entropy of fusion as metallic compounds, as both retain a similar degree of short-range order across melting. In contrast, the slope of the line fit to the SC-M data in Figure 2 is $41.2 \mathrm{~J} \mathrm{~mol}^{-1} \mathrm{~K}^{-1}$. This reflects the dramatic decrease in short-range order upon 
melting of these semiconducting compounds, which contributes a large configurational entropy of fusion as well as a large electronic entropy of fusion $[3,5,6,9,10]$.

The difference in enthalpies of fusion between systems that maintain semiconductivity and systems that metallize upon melting can be leveraged to predict whether a system will behave as a molten semiconductor. We begin by analyzing the condition of stability for the metallized or semiconductor state of molten systems.

The free energy of mixing $\left(\Delta G_{m i x}\right)$ (see [5] for a reminder on mixing terms) determines the phase diagram of a system and is comprised of enthalpy $\left(\Delta H_{m i x}\right)$ and entropy $\left(\Delta S_{m i x}\right)$ terms:

$$
\Delta G_{m i x}=\Delta H_{m i x}-T \Delta S_{m i x}
$$

We consider a binary mixture that has a congruent-melting solid-state semiconductor compound and ask whether the mixture at the composition of the compound will either retain its semiconducting properties (SC-SC transition, superscript SC) or metallize (SC-M, superscript M) upon melting. The system will behave as a molten semiconductor if its free energy in the molten semiconductor state $\left(\Delta G_{m i x}^{S C}\right)$ is lower than its free energy in the metallized state $\left(\Delta G_{m i x}^{M}\right)$. Consequently, determining whether a system will behave as a semiconductor in the molten state is equivalent to determining the validity of the inequality:

$$
\Delta G_{m i x}^{S C}<\Delta G_{m i x}^{M}
$$

Breaking the free energy into its enthalpic and entropic components leads to:

$$
\Delta H_{m i x}^{S C}-T \Delta S_{m i x}^{S C}<\Delta H_{m i x}^{M}-T \Delta S_{m i x}^{M}
$$

Rearranging, we can derive a conclusion about the necessary magnitude of the entropy of mixing for a system to behave as a molten semiconductor:

$$
\Delta S_{m i x}^{S C}>\Delta S_{m i x}^{M}+\frac{\left(\Delta H_{m i x}^{S C}-\Delta H_{m i x}^{M}\right)}{T}
$$


We can understand each term in Eq. 4 as a change in entropy that occurs upon mixing. The left hand term $\Delta S_{\text {mix }}^{S C}$, the entropy of mixing of the molten semiconductor state, describes the entropy associated with mixing of end members for the hypothetical molten semiconductor state of the system. The first term on the right hand side, $\Delta S_{m i x}^{M}$, the entropy of mixing of the metallized molten state, describes the entropy associated with mixing of end members for the hypothetical metallized molten state of the system. Both terms can include entropy associated with atomic disorder (configurational entropy), vibrational entropy, electronic entropy, and more. The second term on the right hand side of Eq. 4 also has the dimension of entropy, representing the bonding energy due to the modification of the local ordering necessary for a semiconductorto-metal transition in the molten state. The enthalpy of mixing of both the hypothetical semiconductor and metallized state are negative. Since the reduction in the short-range order of the system upon metallization will lead to a reduction in the magnitude of the enthalpy, the following inequality holds:

$$
\Delta H_{m i x}^{S C}<\Delta H_{m i x}^{M}
$$

i.e. the second term on the right side of Eq. 4 will always be negative.

Our previous work showed that electronic entropy of mixing comprises the majority of the total entropy of mixing for molten semiconductors [5]. Indeed, molten semiconductors have substantial short-range order, and consequently do not exhibit large configurational entropies of mixing. In addition, the vibrational component of the entropy of mixing has been shown to be minimal [5]. Consequently, the electronic entropy of mixing dominates the total entropy of mixing for these systems.

Thus, replacing the entropy of mixing of the semiconducting state $\left(\Delta S_{m i x}^{S C}\right)$ with the electronic entropy of mixing $\left(\Delta S_{e}\right)$ of the semiconducting state in Eq. 5 leads to: 


$$
\Delta S_{e}>\Delta S_{m i x}^{M}+\frac{\left(\Delta H_{m i x}^{S C}-\Delta H_{m i x}^{M}\right)}{T}
$$

Eq. 6 indicates that a system will behave as a molten semiconductor if the electronic entropy of mixing exceeds the entropy of mixing of the metallized state less the reduction in the magnitude of enthalpy associated with the loss of short-range order that would occur upon metallization.

The first term to the left of Eq. $6 ., \Delta S_{m i x}^{M}$, is dominated by configurational entropy since systems metallized in the molten state have lower short-range order than their semiconductor counterparts. Consequently, the maximum entropy of mixing of a metallized state approximates the ideal entropy of mixing.

In this framework, electronic transitions for a melt will happen upon a specific redistribution of the bonding energy. Upon metallization, electronic entropy of mixing is reduced in favor of configurational entropy to satisfy the stability condition. For systems that undergo a semiconductor-to-semiconductor transition, this redistribution does not occur and the stability condition is satisfied by a retention of semiconducting properties, which maximizes, in particular, electronic entropy of mixing.

Historically, a thermodynamic stability analysis as performed above was limited due to the inability to predict or measure electronic entropy $\left(\Delta S_{e}\right)$ in Eq. 6 . The connection proposed recently in reference 5 between empirically accessible electronic properties of a system and the electronic state entropy of mixing, reproduced herein as Eq. 7, now allows further analysis.

$$
\Delta S_{e}=\frac{\sigma}{\mu} \alpha-x_{A} S_{e}^{A}-x_{B} S_{e}^{B}
$$

In Eq. 7, $x_{A}$ is the concentration of A, $x_{B}$ is the concentration of $\mathrm{B}, S_{e}^{A}$ is the absolute electronic entropy of pure A, and $S_{e}^{B}$ is the absolute electronic entropy of pure B, and other parameters are 
empirical data on the conductivity $(\sigma)$, thermopower $(\alpha)$, and mobility $(\mu)$ of the molten A-B mixture.

We test the stability condition of Eq. 6 to predict molten semiconductivity by analyzing the tellurium-thallium system. The phase diagram of the Te-Tl system is presented in Figure 3. A single congruent melting compound, $\mathrm{TeTl}_{2}$, is exhibited, as well as a miscibility gap in the thallium-rich portion of the phase diagram.

The regions of the phase diagram shown in Figure 3, and all further figures, describe the state of the molten phase at $770 \mathrm{~K}$. Region 1 indicates the semiconducting molten phase. Region 2 indicates a two-phase region. Region 3 indicates a metallized molten phase.

Figure 4 shows the electronic entropy of mixing as predicted by Eq. $7\left(\Delta S_{e}\right.$, solid $)$ vs. the ideal entropy of mixing ( $\Delta S_{\text {ideal }}$, dashed) for the Tl-Te system at $770 \mathrm{~K}$ using the empirical conductivity $(\sigma)$, thermopower $(\alpha)$, and mobility $(\mu)$ provided by Cutler $[5,11,12]$. For this system, the electronic entropy of mixing exceeds the ideal configurational entropy of mixing in the region of the miscibility gap (region 2). Consequently, and surprisingly without a full description of the enthalpy of mixing, Eq. 6 shows that the high electronic entropy of mixing in the immiscible state drives the thermodynamics of the system such that immiscibility (and molten semiconductivity) is energetically preferred over the unsegregated metallized state. Of particular note is that this electronic entropy of mixing itself allows the delimitation of the range of immiscibility present in the system, as the electronic entropy of mixing in the shaded region is a tie-line between the entropy of mixing of the two segregated phases.

Leveraging empirical data for the enthalpy of mixing of the Te-Tl system and the modified Richard's rule discussed above, we can further approximate the right hand side of Eq. 6 
as a function of composition for the Te-Tl system. To simplify the analysis we will define the right hand side of Eq. 6 as $\xi$ :

$$
\xi=\Delta S_{m i x}^{M}+\frac{\left(\Delta H_{m i x}^{S C}-\Delta H_{m i x}^{M}\right)}{T}
$$

As derived in the Appendix, the difference in enthalpies of fusion of the molten semiconductor and the metallized molten state at a given temperature can be estimated from the data presented in Figure 2. Because the thermodynamics of the end-members (Te and $\mathrm{Tl}$ ) and the solid-state compound $\left(\mathrm{TeTl}_{2}\right)$ have no influence on the evaluation of Eq. 8 (i.e. are the same whether a system undergo a SC-SC or SC-M transition), the difference in enthalpies of mixing at the compound composition for the molten state translates into a difference in enthalpy of fusion (Eq. 9). Eq. 9 holds explicitly true at the melting temperature, but is approximately true for temperatures near melting (the contributions of differing heat capacities of the molten semiconductor and molten metallized states are minor compared to the difference in enthalpy of fusion).

$$
\Delta H_{m i x}^{S C}-\Delta H_{m i x}^{M} \approx \Delta H_{f}^{S C}-\Delta H_{f}^{M}
$$

Leveraging Eq. 9 and approximating $\Delta S_{\text {mix }}^{M}$ as the ideal entropy of mixing $\left(\Delta S_{\text {ideal }}\right)$ we can further define $\xi$ as:

$$
\xi=\Delta S_{\text {ideal }}^{M}+\frac{\left(\Delta H_{f}^{S C}-\Delta H_{f}^{M}\right)}{T}
$$

The enthalpy of fusion data $\left(\Delta H_{f}^{S C}, \Delta H_{f}^{M}\right)$ are predicted by the line of best fit from Figure 2 (analogous to Richard's rule), and Figure 5 compares the variation of the electronic entropy of mixing of the semiconducting state $\left(\Delta S_{e}\right.$, solid) with $\xi$ (dashed).

It can be clearly seen that $\Delta S_{e}$ exceeds $\xi$ for the entirety of the composition range with the exception of the thallium-rich portion of the phase diagram (region 3). Comparing to the phase diagram, we note that the region of Figure 5 where Eq. 6 is satisfied (region 1) 
corresponds to a semiconducting molten phase while the region of Figure 5 where Eq. 6 is not satisfied (region 3) corresponds to the molten metallic phase (as validated by empirical measurements of the electronic properties by Cutler [12]) on the thallium-rich side of the miscibility gap.

We can broadly understand the presence of a molten semiconductor phase as a consequence of the high electronic entropy of mixing of a melt inherited from the strong shortrange order necessary for semiconducting properties. The electronic entropy of mixing, combined with the negative enthalpies of mixing associated with retention of short-range order, lowers the free energy of the system and renders the semiconducting state more energetically favorable than a disordered, and consequently low electronic entropy of mixing, metallized system.

The key features of the phase diagrams shown in Figure 1 and their link to the electronic behavior of the molten phase can be explained through this lens. The presence of a congruent melting semiconductor compound allows for a relative continuity of short-range order (and hence electronic properties and the electronic entropy of mixing) across the liquidus. Immiscibility is present in systems exhibiting molten semiconductivity (SC-SC) due to the energetic stability of the ordered, high electronic state entropy of mixing in the vicinity of the composition of the congruent melting compound. Conversely, for systems that metallize upon melting (SC-M), the stability condition favors configurational disorder, and consequently a continuous and homogenous phase region is possible across the entire range of composition.

More generally, Eq. 6 provides an estimate of the minimum electronic entropy required to achieve a molten semiconductor state. Through the model shared by Rinzler and Allanore this 
correspondingly bounds the electronic properties (thermopower, electronic conductivity, and mobility) of molten semiconductor systems for a given set of end member elements.

\section{Conclusion}

Thanks to the use of the recently proposed quantification of electronic state entropy of the molten state, the thermodynamic origin of a correlation between certain features of phase diagrams and the electronic properties of molten semiconductor systems has been proposed. It is found that being able to quantify electronic state entropy allows the definition of conditions for miscibility in the molten state, which in turns enables the evaluation of the range of electronic properties that this state can exhibit. Electronic state entropy is also shown to be pertinent in explaining the deviation from Richard's rule for certain systems, and is therefore put forth as an important thermodynamic function for understanding solid-liquid phase transitions. 


\section{Appendix}

The enthalpy of fusion of a system undergoing a semiconductor-to-semiconductor (SC-SC) transition at melting is:

$$
\Delta H_{f}^{S C}=H_{L}^{S C}-H_{S}^{S C},
$$

where $H_{L}^{S C}$ and $H_{S}^{S C}$ are the enthalpies of the liquid and solid state at the melting temperature.

Similarly, we can define the enthalpy of fusion of a system undergoing a semiconductor-to-metal (SC-M) transition:

$$
\Delta H_{f}^{M}=H_{L}^{M}-H_{S}^{M}
$$

In our analysis, the solid state remains unchanged, hence:

$$
H_{S}^{S C}=H_{S}^{M}
$$

Thus, the difference in magnitude of the enthalpies of fusion of the semiconductor (SC) and metallic (M) molten phase is:

$$
\Delta H_{f}^{S C}-\Delta H_{f}^{M}=H_{L}^{S C}-H_{L}^{M}
$$

The enthalpies are defined in terms of mixing and mechanical terms:

$$
H_{L}^{S C}=\Delta H_{m i x}^{S C}+H_{m e c h}^{S C}
$$

and

$$
H_{L}^{M}=\Delta H_{m i x}^{M}+H_{m e c h}^{M}
$$

However, because in this analysis the end members are the same in both the semiconductor and metallic molten states, the mechanical terms are equivalent:

$$
H_{m e c h}^{S C}=H_{m e c h}^{M}=x_{A} H_{A}+x_{B} H_{B}
$$

where $x_{A}$ and $x_{B}$ are the concentrations of end-members $\mathrm{A}$ and $\mathrm{B}$ and $H_{A}$ and $H_{B}$ are the absolute enthalpies of the end-members A and B (for a two component system A-B).

Thus, the difference in enthalpies of the semiconducting and metallic phases is: 


$$
H_{L}^{S C}-H_{L}^{M}=\Delta H_{m i x}^{S C}-\Delta H_{m i x}^{M}
$$

and we can thus equate the difference in enthalpies of fusion to the difference in enthalpies of mixing:

$$
\Delta H_{f}^{S C}-\Delta H_{f}^{M}=\Delta H_{m i x}^{S C}-\Delta H_{m i x}^{M}
$$




\section{References}

[1] A. F. Ioffe and A. R. Regel, Progress in Semiconductors, Wiley, New York, NY, 1960.

[2] N. F. Mott and E. A. Davis, Electronic Processes in Non-Crystalline Materials, Clarendon Press, Oxford, UK, 1971.

[3] D. P. Belotskii and O. N. Manik, On the interrelation between electronic properties and structure of thermoelectric material melts and the state diagrams - 5. classification of electronic semiconductor melts, J. Thermoelectr. 1 (2004), pp. 32-47.

[4] B. Fultz, Phase Transitions in Materials, Cambridge University Press, Cambridge, UK, 2014.

[5] C. Rinzler and A. Allanore, Connecting electronic entropy to empirically accessible electronic properties in high temperature systems, Philos. Mag. 96 (2016), pp. 30413053.

[6] V. M. Glazov, Liquid Semiconductors, Plenum Press, New York, NY, 1969.

[7] T. Iida and R. Guthrie, The Thermophysical Properties of Metallic Liquids, Oxford University Press, Oxford, UK, 2015.

[8] D. Gaskel, Introduction to the Thermodynamics of Materials, 5th ed., CRC Press, Boca Raton, FL, 1995.

[9] A. R. Regel, V. M. Glazov, and A. A. Aivazov, Calculation of components of fusion entropy of some semiconducting compounds, Sov. Phys. Semicond. 8 (1975), pp. 13981401.

[10] D. Adler, Physics of Disordered Materials, Plenum Press, New York, NY, 1985.

[11] M. Cutler and C. E. Mallon, Thermoelectric properties of liquid semiconductor solutions of thallium and tellurium, J. Appl. Phys. 36 (1965), pp. 201-205.

[12] M. Cutler, Liquid Semiconductors, Academic Press, New York, NY, 1977.

[13] H. Okamoto, Te-tl (tellurium-thallium), J. Phase Equilibria 12 (1991), pp. 507-508. 


\section{Acknowledgements}

This work was supported by the AFOSR under Grant FA9550-15-1-0046. We would like to

acknowledge the role of the Fannie and John Hertz Foundation for support of the researchers and Dr. Ali Sayir and the AFOSR for their financial support. 


\section{Figure Captions}

Figure 1. Nominal phase diagrams of systems undergoing a semiconductor-to-semiconductor (SC-SC, left) and semiconductor-to-metal (SC-M, right) transition on melting. SC-SC systems tend to exhibit a congruent melting compound $(\mathrm{AB})$ and one or more miscibility gaps (shown as a dashed line). SC-M systems may exhibit a congruent melting compound, but do not exhibit liquid phase miscibility gaps. Thatched shaded regions behave as semiconductor systems and solid shaded regions behave as metallic systems. B is assumed to be metallic.

Figure 2. Enthalpy of fusion $\left(\Delta \mathrm{H}_{\mathrm{f}}\right)$ vs. melting temperature $\left(\mathrm{T}_{\mathrm{m}}\right)$ for systems that exhibit semiconductor-to-semiconductor (SC-SC) transitions at melting (circles) and systems that exhibit semiconductor-to-metal (SC-M) transitions at melting (diamonds). Calculated from measurements reported in [3]. The slopes of best line of fit are $10.0 \mathrm{~J} \mathrm{~mol}^{-1} \mathrm{~K}^{-1}$ and $41.2 \mathrm{~J} \mathrm{~mol}^{-1}$ $\mathrm{K}^{-1}$ respectively. The slope of best fit for the SC-SC systems is similar to the slope predicted by Richard's rule for metallic alloy systems (a slope of 9.6 $\mathrm{J} \mathrm{mol}^{-1} \mathrm{~K}^{-1}$ ) [8].

Figure 3. Phase diagram of the tellurium-thallium system as reported by Okamoto [13]. Region 1 indicates the semiconducting molten phase. Region 2 indicates a two-phase region. Region 3 indicates a metallized molten phase.

Figure 4. $\Delta S_{e}$ (solid) as calculated by Rinzler and Allanore and the ideal entropy of mixing ( $\Delta S_{\text {ideal }}$ dashed) for Te-Tl at $770 \mathrm{~K}$ [5]. The miscibility gap (region 2 ) is shaded.

Figure 5. $\Delta S_{e}$ (solid) as calculated by Rinzler and Allanore and the $\xi$ parameter from Eq. 10 (dashed) for Te-Tl at $770 \mathrm{~K}$. The $\xi$ parameter does not have a physical meaning in the presence of a miscibility gap (the shaded region) [5]. 


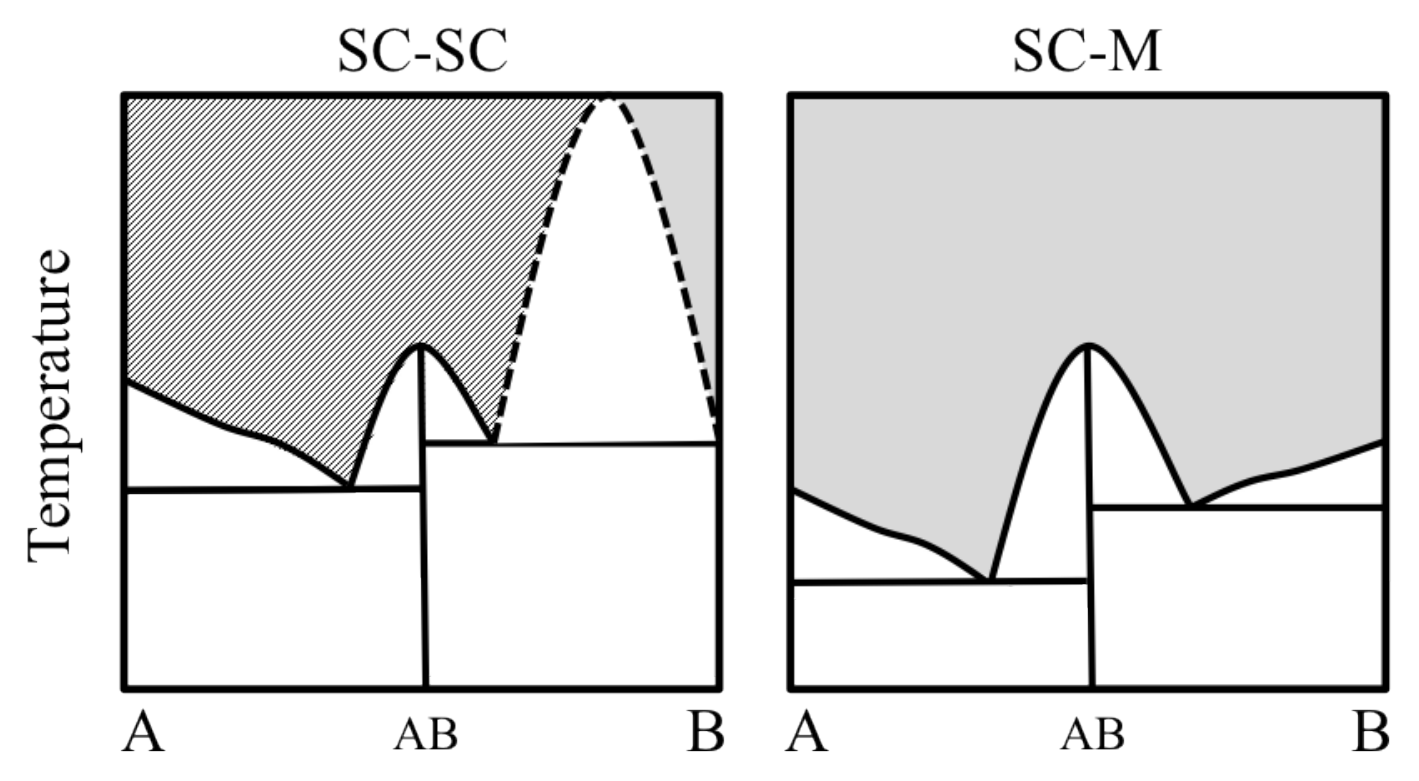

Fig 1 


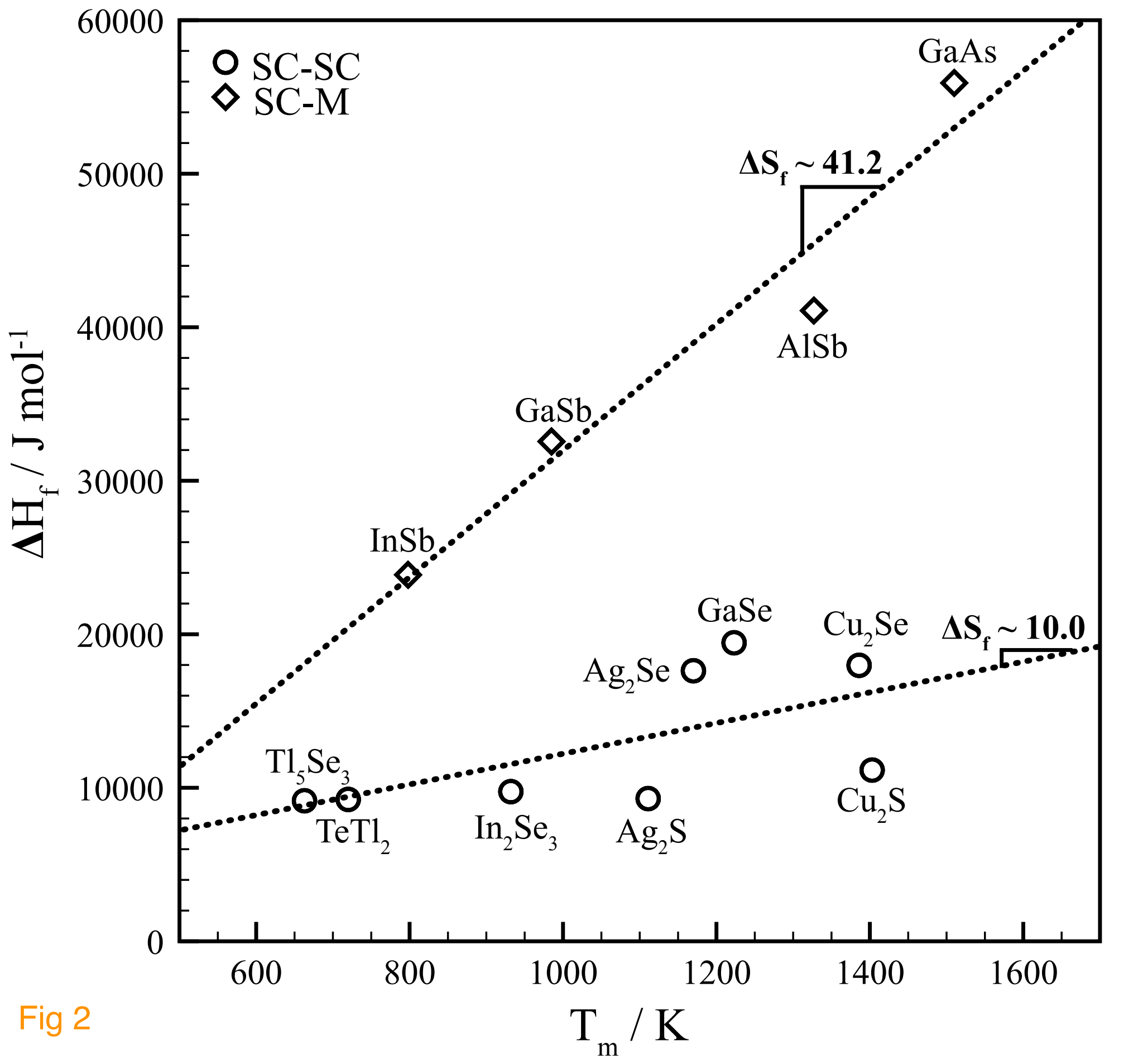




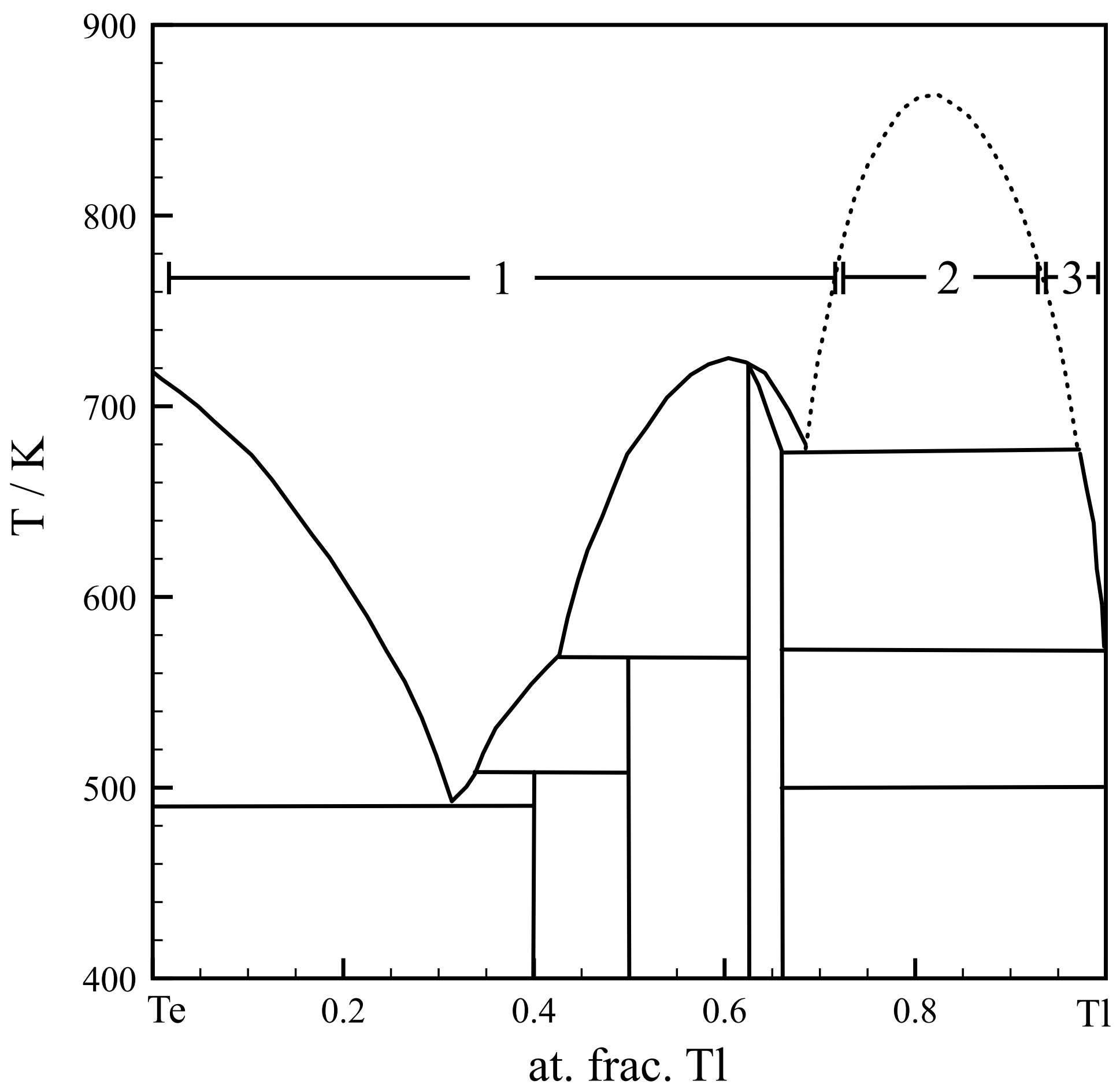


